\title{
Correction to: Estimating the leaf area index of bell peppers according to growth stage using ray-tracing simulation and a long short-term memory algorithm
}

\author{
Joon Woo Lee ${ }^{1}$. Woo Hyun Kang ${ }^{2} \cdot$ Taewon Moon $^{2} \cdot$ Inha Hwang $^{2}$ · Dongpil Kim² · Jung Eek Son ${ }^{2,3}$
}

Published online: 17 March 2020

(c) Korean Society for Horticultural Science 2020

\section{Correction to:}

\section{Horticulture, Environment, and Biotechnology}

https://doi.org/10.1007/s13580-019-00214-9

The original version of this article unfortunately contained a mistake. The affiliation of Joon Woo Lee was changed during the review process. The corrected affiliations are given below.

${ }^{1}$ Department of Smart Agriculture, Jeonju University, Jeonju, 55069, Korea

${ }^{2}$ Department of Plant Science, Seoul National University, Seoul 08826, Korea

${ }^{3}$ Research Institute of Agriculture and Life Sciences, Seoul National University, Seoul 08826, Korea

The original article can be found online at https://doi.org/10.1007/ s13580-019-00214-9.

Jung Eek Son

sjeenv@snu.ac.kr

1 Department of Smart Agriculture, Jeonju University, Jeonju 55069, Korea

2 Department of Plant Science, Seoul National University, Seoul 08826, Korea

3 Research Institute of Agriculture and Life Sciences, Seoul National University, Seoul 08826, Korea
Publisher's Note Springer Nature remains neutral with regard to jurisdictional claims in published maps and institutional affiliations. 